\title{
Observation of Double Excitations in the Resonant Inelastic X-ray Scattering of Nitric Oxide
}

Adam E. A. Fouda ${ }^{\text {a) }}$, Linsey C. Seitz ${ }^{\mathrm{b}), \mathrm{c})}$, Dirk Hauschild ${ }^{\mathrm{b}), \mathrm{d}), \mathrm{e}}$, Monika Blum ${ }^{\mathrm{e}, \mathrm{f}), \mathrm{g})}$, Wanli Yang ${ }^{\mathrm{f}}$,

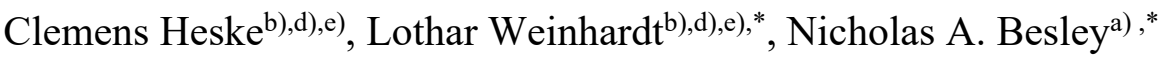

a) School of Chemistry, University of Nottingham, University Park, Nottingham, NG7 2RD, UK

b) Institute for Photon Science and Synchrotron Radiation (IPS), Karlsruhe Institute of Technology (KIT), Hermann-v.-Helmholtz-Platz 1, 76344 Eggenstein-Leopoldshafen, Germany

c) Department of Chemical and Biological Engineering, Northwestern University, 2145 Sheridan Road, Evanston, IL 60208, USA

d) Institute for Chemical Technology and Polymer Chemistry (ITCP), Karlsruhe Institute of Technology (KIT), Engesserstr. 18/20, 76128 Karlsruhe, Germany

e) Department of Chemistry and Biochemistry, University of Nevada, Las Vegas (UNLV), 4505 Maryland Parkway, Las Vegas, NV 89154-4003, USA

f) Advanced Light Source, Lawrence Berkeley National Laboratory, 1 Cyclotron Road, Berkeley, CA 94720, USA

g) Chemical Sciences Division, Lawrence Berkeley National Laboratory, 1 Cyclotron Road, Berkeley, CA 94720, USA

*Corresponding authors: Email: Nick.Besley@nottingham.ac.uk, Lothar.Weinhardt@kit.edu

Manuscript accepted in The Journal of Physical Chemistry Letters 


\begin{abstract}
The nitrogen K-edge resonant inelastic x-ray scattering (RIXS) map of nitric oxide (NO) has been measured and simulated to provide a detailed analysis of the observed features. High-resolution experimental RIXS maps were collected using an in situ gas flow cell and a high-transmission soft X-ray spectrometer. Accurate descriptions of the ground, excited, and core-excited states are based upon restricted active space self-consistent-field calculations using second order multiconfigurational perturbation theory. The nitrogen K-edge RIXS map of NO shows a range of features that can be assigned to intermediate states arising from $1 \mathrm{~s} \rightarrow \pi^{*}$ and $1 \mathrm{~s} \rightarrow \mathrm{Rydberg}$ excitations; additional bands are attributed to doubly excited intermediate states comprising $1 \mathrm{~s} \rightarrow \pi^{*}$ and $\pi \rightarrow \pi^{*}$ excitations. These results provide a detailed picture of RIXS for an open-shell molecule and an extensive description of the core-excited electronic structure of NO, an important molecule in many chemical and biological processes.
\end{abstract}

\title{
TOC Graphic
}

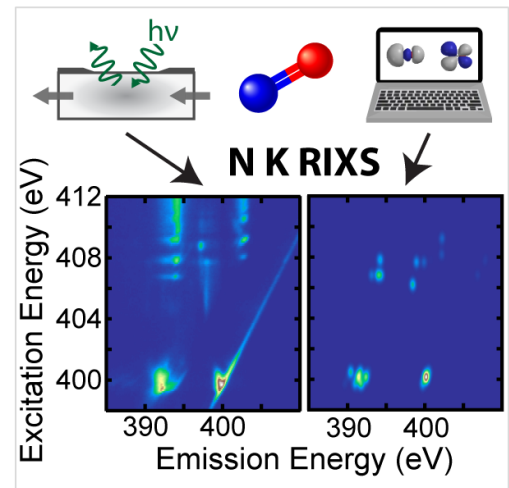


Advances in synchrotron-based light sources have led to the emergence of x-ray spectroscopic techniques as powerful tools for the study of molecular structure and ultrafast molecular dynamics. ${ }^{1-6}$ Among these techniques, resonant inelastic (soft) $\mathrm{x}$-ray scattering (RIXS) is a twophoton technique, wherein the initial state of the system is excited to an intermediate core-excited state and the emission from the subsequent de-excitation is measured. ${ }^{7}$ RIXS provides a wealth of information on aspects such as the electronic structure, screening effects, non-adiabatic processes, and ultrafast dynamics that occur within the lifetime of the intermediate state..$^{2-4,8,9}$ Using a suitable high-transmission spectrometer, ${ }^{10}$ the emission can be measured in a densely spaced series of excitation energies, leading to a "RIXS map" that shows the intensity as a function of the excitation and emission energies. ${ }^{10,11}$

RIXS maps have been reported for a variety of systems, including molecules in the gas phase. For example, gas-phase water has been studied in detail, revealing the occurrence of core-excited ultrafast dissociation. ${ }^{4,12-14}$ Furthermore, diatomic molecules, such as molecular nitrogen, carbon monoxide, and molecular oxygen, have been studied using RIXS. A resonant x-ray emission study of molecular nitrogen showed the presence of a parity selection rule, ${ }^{15}$ and Rubensson and coworkers found that, for near-threshold states, the core-excited Rydberg electron acts as a spectator in the resonant emission process. ${ }^{16}$ Screening effects in resonantly excited emission spectra of carbon monoxide have been discussed in detail, ${ }^{17}$ and the presence of dark two electron-one photon final states attained by non-adiabatic dynamics driven by Coulomb coupling has been observed. ${ }^{18}$ Symmetry effects upon core-hole excitation have been investigated for molecular oxygen, ${ }^{19,20}$ and a vibrational RIXS study observed a spatial quantum beat effect arising from interference between two wave packets with different momenta for dissociative core-excited states. ${ }^{21}$ The $\mathrm{x}$-ray 
absorption (XAS) and resonant Auger spectra for the $1 \mathrm{~s} \rightarrow \sigma^{*}$ transition in molecular oxygen have been reported, and the theoretical analysis suggests a complex picture with multiple crossings of the potential energy curves for the core-excited states and a breakdown of the Born-Oppenheimer approximation..$^{22}$

The focus of this work is nitric oxide (NO), a stable open-shell molecule with an electronic configuration $\left(1 \mathrm{so}^{2}\right)^{2}\left(1 \mathrm{~s}_{\mathrm{N}}\right)^{2}(3 \sigma)^{2}(4 \sigma)^{2}(5 \sigma)^{2}\left(\pi_{\mathrm{x}}\right)^{2}\left(\pi_{\mathrm{y}}\right)^{2}\left(\pi_{\mathrm{x}}{ }^{*}\right)^{1}\left(\pi_{\mathrm{y}}{ }^{*}\right)^{0}(3 \mathrm{~s})^{0}\left(3 \mathrm{p}_{\pi \mathrm{x}}\right)^{0}\left(3 \mathrm{p}_{\pi \mathrm{y}}\right)^{0}\left(3 \mathrm{p}_{\sigma}\right)^{0}$ and a $X^{2} \Pi$ ground state. It is important in many chemical and biological processes, including the formation of smog and acid rain, as well as the depletion of ozone in the atmosphere. NO also plays a role in the control of blood circulation, nerve transmission, and the function of the immune system. ${ }^{23}$ The UV photoemission spectrum of NO has been studied in detail, revealing that the $\pi \rightarrow \pi^{*}$ transition leads to a set of valence excited states including $B^{2} \Pi, L^{\prime} \Phi$, and $L^{2} \Pi$, while excitation to the $3 \mathrm{~s}$ and 3p Rydberg orbitals gives $A^{2} \Sigma^{+}, C^{2} \Pi$, and $D^{2} \Sigma^{+}$states. ${ }^{24-26}$ Furthermore, a significant lengthening of the bond occurs for $\pi \rightarrow \pi^{*}$ excitations, while excitation to the Rydberg orbitals leads to a shortening of the bond. ${ }^{26}$

In comparison, there have been relatively few studies focused on the core-excited states of NO. The $\mathrm{x}$-ray absorption spectrum of NO at the nitrogen K-edge has been measured using electron energy-loss spectroscopy ${ }^{27,28}$ and also in a high-resolution photoionization study. ${ }^{29}$ The spectrum is dominated by an intense band at approximately $400 \mathrm{eV}$, arising from the $1 \mathrm{~s}_{\mathrm{N}} \rightarrow \pi^{*}$ transitions, with weaker bands at higher energies due to transitions to Rydberg states. The $1 \mathrm{~s}_{\mathrm{N}}^{-1} \pi^{* 2}$ electron configuration gives rise to one quartet and three doublet electronic states, namely ${ }^{4} \Sigma^{-},{ }^{2} \Sigma^{-},{ }^{2} \Delta$, and ${ }^{2} \Sigma^{+}$. However, only transitions to the doublet states make a significant contribution to the observed 
band. The orbital occupancies of these states are illustrated in Figure S1. There has been some debate regarding the energy ordering of the three doublet states, but high-level calculations suggest that the order is, from low to high energy, ${ }^{2} \Delta,{ }^{2} \Sigma^{-},{ }^{2} \Sigma^{+} .{ }^{29}$ It has also been noted that the potential energy surfaces for the ${ }^{2} \Delta$ and ${ }^{2} \Sigma^{-}$states are similar, while the surface for the ${ }^{2} \Sigma^{+}$state is different. ${ }^{30}$ There is a significant lengthening of the bond in these core-excited states relative to the ground state, and vibrational structure is observed both in the nitrogen and oxygen K-edge XAS spectra of NO. ${ }^{31,32}$ Theoretical simulations of the absorption and de-excitation spectra associated with the $1 \mathrm{~s}_{\mathrm{N}} \rightarrow \pi^{*}$ transitions using potential energy surfaces determined from the multiconfiguration reference coupled electron pair approximation give good agreement with the experimental coreexcitation energies. ${ }^{30}$ Other studies have measured the angular distribution of the nitrogen K-edge photoelectron ionization and Auger decay of NO, which show that interference between excitation and decay channels of different symmetries plays a significant role..$^{33,34}$

In this Letter, the nitrogen K-edge RIXS map of the NO molecule is presented, providing a detailed picture of RIXS in an open-shell molecule and an extensive description of the core-excited electronic structure of NO. This, in turn, provides insight into the processes that occur on the lifetime of the core-excited states. The measured RIXS map is interpreted using simulated maps based upon multiconfigurational perturbation theory calculations, allowing the observed features to be assigned to electronic states. The results reveal the presence of distinct features in the RIXS spectra that can be attributed to the formation of doubly excited states.

The measured nitrogen K-edge RIXS map for gas-phase NO is shown in Figure 1 (a), with detailed regions shown in Fig. 1 (b). A simulated map based upon ab initio calculations is depicted in 
Figure 1 (c). Different detail regions of the map are labelled i-iii and also indicated in the measured and calculated maps. Also shown is the non-resonant x-ray emission spectrum (Figure 1 (d)), which will be discussed in more detail below. The electronic structure of NO represents a challenge for electronic structure theory, owing to the multiconfigurational nature of the wave functions and the necessity to describe both valence states and diffuse Rydberg states. To achieve an accurate description of the ground, excited, and core-excited states, the simulated map is based upon restricted active space self-consistent-field (RASSCF) ${ }^{35}$ calculations, with the state energies improved via addition of dynamical correlation using restricted active space second order perturbation theory (RASPT2) ${ }^{36}$ and the RIXS intensity evaluated using the Kramers-Heisenberg formalism. ${ }^{37-40}$ The simulated map provides a good foundation for a detailed analysis of the observed features.

Before analyzing the RIXS map, we will discuss the non-resonant emission spectrum [Figure 1 (d)]. The experimental spectrum (solid purple line) shows strong peaks at $\sim 402.5$ and $\sim 394.5 \mathrm{eV}$, the latter with a shoulder at lower energy. Closer inspection of the spectrum shows weaker features at $389 \mathrm{eV}$ and in the region $397-400 \mathrm{eV}$, which can be observed more clearly in the magnified spectrum (15x, dark green sections). Simulations of the non-resonant emission spectrum based on equation-of-motion coupled cluster calculations (see Supporting Information) show that the peak at $402.5 \mathrm{eV}$ is attributed to the singly occupied $\pi^{*}$ orbital, while the peak at $394.5 \mathrm{eV}$ and its shoulder correspond to the $5 \sigma$ and $\pi$ orbitals, respectively. The feature at $389.0 \mathrm{eV}$ can be attributed to emission from the $4 \sigma$ orbital, although the intensity of this transition is very low, in accordance with the dipole selection rules. The calculated emission spectrum shows no evidence of transitions in the $397-400 \mathrm{eV}$ energy range. Similar "satellites" have been observed previously for other 
small molecules, e.g., gas phase water, and attributed to double excitations. ${ }^{41}$ We have thus computed the emission spectra for the case when the core-ionization is accompanied by $\pi \rightarrow \pi^{*}$ and $5 \sigma \rightarrow \pi^{*}$ excitations, i.e. valence electronic configurations of $(5 \sigma)^{2}(\pi)^{3}\left(\pi_{\mathrm{x}}^{*}\right)^{1}\left(\pi_{\mathrm{y}}{ }^{*}\right)^{1}$ and $(5 \sigma)^{1}(\pi)^{4}\left(\pi_{\mathrm{x}}\right)^{1}\left(\pi_{\mathrm{y}}\right)^{1}$, respectively. The calculation results are shown as blue and red bars in Figure 1(d) and in more detail in Figures S2 and S3. The doubly excited states including a $\pi \rightarrow \pi^{*}$ transition lead to $5 \sigma$ emission at 397.7 and $398.0 \mathrm{eV}$, which is consistent with the weak bands observed in the experimental spectrum. Similarly, $\pi$ and $\pi^{*}$ emission peaks for doubly excited final states are expected at 392.5, 395.5, and $402.3 \mathrm{eV}$, overlapping with the strongest features for the core-ionized state.

We now turn back to the RIXS maps in Figure 1. Region $\mathrm{i}$ is dominated by two intense and broad features at an absorption energy of $\sim 400 \mathrm{eV}$ and with emission energies of $\sim 400 \mathrm{eV}$ and $\sim 392 \mathrm{eV}$, respectively. This region arises from the $1 \mathrm{~s}_{\mathrm{N}} \rightarrow \pi^{*}$ excitation, which gives the $\left(1 \mathrm{~s}_{\mathrm{N}}\right)^{1}\left(\pi^{*}\right)^{2}$ electron configuration leading to ${ }^{2} \Delta,{ }^{2} \Sigma^{-}$, and ${ }^{2} \Sigma^{+}$intermediate states. Note that we do not consider the ${ }^{4} \Sigma^{-}$ state due to its vanishing intensity. The calculated excitation energies for these states are given in Table 1, predicting an energy ordering consistent with previous multiconfiguration reference coupled electron pair approximation calculations. ${ }^{30}$ The calculated energies are close to those derived from the experimental spectra. ${ }^{29}$ The feature at an emission energy of $\sim 400 \mathrm{eV}$ corresponds to the participant decay involving the $\pi^{*}$ orbital and lies on or close to the diagonal line of elastically scattered photons at equal excitation and emission energies (i.e., the Rayleigh line). It extends to lower emission energies due to vibrational excitations that lead to a vibrational envelope (not resolved into individual peaks in the present experiment). 


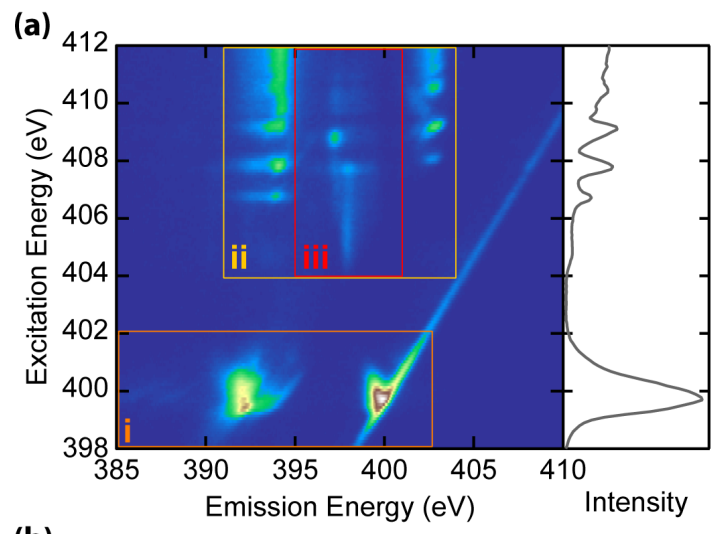

(b)
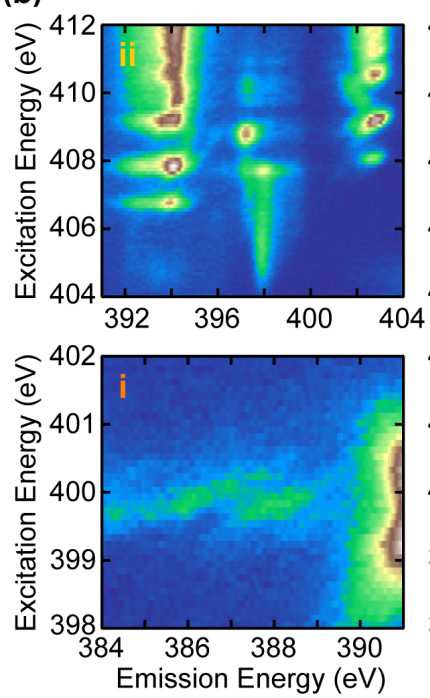

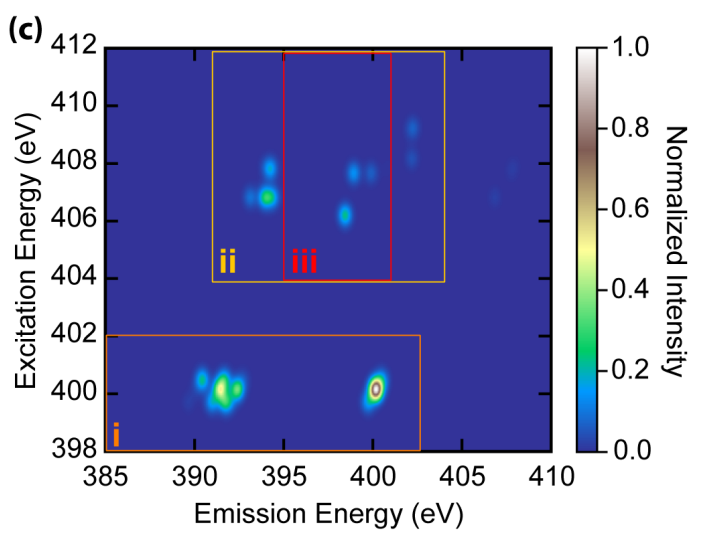

(d)

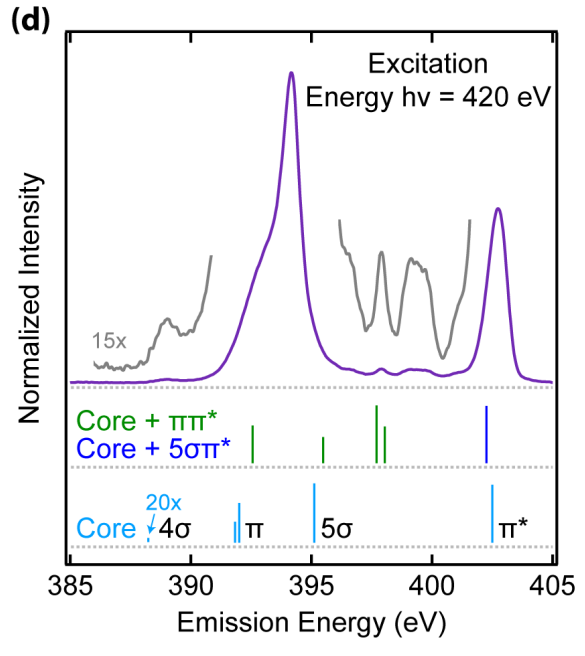

Figure 1: (a) Experimental resonant inelastic x-ray scattering (RIXS) map at the nitrogen K-edge for NO gas, with various highlighted regions (labeled i-iii) shown in larger detail in (b). (c) Simulated nitrogen K-edge RIXS map. (d) Experimental non-resonant x-ray emission spectrum with simulated peak intensities shown below. The molecular orbitals are shown pictorially in the Supporting Information.

The feature at an emission energy of $\sim 392 \mathrm{eV}$ exhibits a rich substructure that is also reflected in the simulations. This substructure can be largely attributed to the energy separation of the intermediate and final states. In total, six spectral contributions, arising from emission from $\pi$ and $5 \sigma$ orbitals for the three $\left(1 \mathrm{~s}_{\mathrm{N}}\right)^{1}\left(\pi^{*}\right)^{2}$ states, are predicted by the simulation of that region. The 
corresponding emission energies are also given in Table 1. We find the emission energy for transitions involving the $\pi$ orbital to be greater than for those involving the $5 \sigma$ orbital for all three of the intermediate states. This comparison suggests that the presence of an additional electron in the $\pi^{*}$ orbital leads to an increase in repulsion for the electrons in the $\pi$ orbital relative to the $5 \sigma$ orbital. While the qualitative agreement between simulation and experiment is reasonable, the experiment shows further fine structure and broadening that is not reflected in the simulation. It can be attributed to vibrational coupling and nuclear dynamics on the timescale of the $\mathrm{x}$-ray emission process. We note that this discussion underlines the need for a multiconfigurational description of the electronic structure, since single determinant-based methods do not adequately describe the energy separation between the involved states. On the low energy side of the $\pi$ and $5 \sigma$ emissions, the experimental map exhibits some weak intensity in the $384-389 \mathrm{eV}$ emission energy range, which is magnified in the lower left panel of Figure 1 (b). We attribute this intensity to the weak $4 \sigma$ transitions, which will be split due to the ${ }^{2} \Delta,{ }^{2} \Sigma^{-}$, and ${ }^{2} \Sigma^{+}$intermediate states.

Region ii of the experimental map contains two sets of features that lie at emission energies of $\sim 394$ and $\sim 402 \mathrm{eV}$. These features show a strong intensity variation as a function of excitation energy, with several distinct peaks. The first set of features appears at an excitation energy of 406.8 $\mathrm{eV}$, then again at 407.9 and $409.3 \mathrm{eV}$, and finally continuing with less pronounced variations at higher excitation energies. The second set of features is observed at an excitation energy of 408.1 $\mathrm{eV}$, then at 409.3 and $410.6 \mathrm{eV}$, and then continues with fewer variations at higher excitation energies. The simulated map also predicts peaks in these regions that correspond to intermediate states arising from $1 \mathrm{~s} \rightarrow$ Rydberg excitations. In the simulated map, only excitation to the $3 \mathrm{~s}, 3 \mathrm{p}_{\pi}$ (Rydberg $\mathrm{p}$ orbitals perpendicular to the internuclear axis), and $3 p_{\sigma}$ (Rydberg $\mathrm{p}$ orbital parallel to 
the internuclear axis) was accounted for; consequently, only features in the lower energy portion of Region ii were captured. The region of the experimental map at higher excitation energies will include excitations to Rydberg $3 \mathrm{~d}$ and $6 \sigma$ (also denoted $2 \mathrm{p} \sigma^{*}$ ) orbitals, but inclusion of these orbitals in the simulation results in an active space that is too large. Excitation to the $3 \mathrm{~s}$ and $3 \mathrm{p}$ Rydberg orbitals generates a large number of potential intermediate states. Excitation to the 3s and $3 p_{\sigma}$ orbitals will lead to three ${ }^{2} \Pi$ states and one ${ }^{4} \Pi$ state each, whereas excitation to the $3 p_{\pi}$ orbital will give six doublet states (three ${ }^{2} \Delta$, two ${ }^{2} \Sigma^{+}$and ${ }^{2} \Sigma^{-}$), in addition to two further quartet states. This results in a total of twelve doublet Rydberg intermediate states included in the simulations. Here, we denote the Rydberg states that arise from the $1 \mathrm{~s}_{\mathrm{N}} \rightarrow \phi$ transition as $\mathrm{R}_{\mathrm{i}}(\phi)$, where $\phi$ is a Rydberg orbital. The calculated absorption and emission energies for these states are summarized in Table 1.

The simulated map predicts bands arising from the Rydberg intermediate states consistent with the features observed in experiment. In particular, the series of features at an emission energy of $\sim 394$ $\mathrm{eV}$ is associated with emission from the $5 \sigma$ and $\pi$ orbitals. For this series, the peak at an excitation energy of $406.9 \mathrm{eV}$ corresponds to the intermediate state arising from the $1 \mathrm{~s}_{\mathrm{N}} \rightarrow 3 \mathrm{~s}$ transition, while the feature at an excitation energy of $407.9 \mathrm{eV}$ is predominantly associated with the Rydberg $3 \mathrm{p}_{\pi}$ and Rydberg $3 p_{\sigma}$ intermediate states. Higher excitation energies will lead to higher energy Rydberg states; however, these are not included in the calculations. For the series of features at $\sim 402 \mathrm{eV}$, assigned to the $\pi^{*}$ emission, the calculations show that the first peak at an excitation energy of $\sim 408.1 \mathrm{eV}$ is associated with the Rydberg $3 \mathrm{p}_{\pi}$ and Rydberg $3 \mathrm{p}_{\sigma}$ intermediate states, while, in agreement with the experiment, no emission for the lowest energy Rydberg 3s intermediate state occurs. Other features in the map are also reproduced by the calculations: Notably, the $\pi, 5 \sigma$, and 
$\pi^{*}$ emission lines shift to $\sim 2 \mathrm{eV}$ higher emission energies for the Rydberg intermediate states as compared to the transitions involving $1 \mathrm{~s}_{\mathrm{N}} \rightarrow \pi^{*}$ excitation. This can be attributed to screening effects (i.e., "spectator shifts"). Furthermore, both experiment and simulation show two weak resonances of the Rayleigh line at $\sim 406.8$ and $\sim 407.9 \mathrm{eV}$.

Table 1. Calculated absorption and emission energies (in eV). Numbers in parentheses denote transitions with very low or vanishing intensity. The valence electronic configuration of the intermediate state is given (all of these states have a singly occupied $1 \mathrm{~s}_{\mathrm{N}}$ orbital).

\begin{tabular}{|c|c|c|c|c|}
\hline & \multirow[t]{2}{*}{ Absorption } & \multicolumn{3}{|c|}{ Emission } \\
\hline & & $\pi^{*}$ & $\pi$ & $5 \sigma$ \\
\hline \multicolumn{5}{|l|}{ Region i } \\
\hline${ }^{2} \Delta\left[(5 \sigma)^{2}(\pi)^{4}\left(\pi^{*}\right)^{2}\right]$ & 399.7 & 399.7 & 391.7 & 391.0 \\
\hline${ }^{2} \Sigma^{-}\left[(5 \sigma)^{2}(\pi)^{4}\left(\pi^{*}\right)^{2}\right]$ & 400.2 & 400.2 & 392.4 & 391.4 \\
\hline${ }^{2} \Sigma^{+}\left[(5 \sigma)^{2}(\pi)^{4}\left(\pi^{*}\right)^{2}\right]$ & 400.5 & 400.5 & 391.7 & 390.5 \\
\hline \multicolumn{5}{|l|}{ Region ii } \\
\hline $\mathrm{R}_{1}(3 \mathrm{~s})\left[(5 \sigma)^{2}(\pi)^{4}\left(\pi^{*}\right)^{1}(3 \mathrm{~s})^{1}\right]$ & 406.8 & $(400.8)$ & 394.4 & $394.0,393.1$ \\
\hline $\mathrm{R}_{2}\left(3 \mathrm{p}_{\pi}\right)\left[(5 \sigma)^{2}(\pi)^{4}\left(\pi^{*}\right)^{1}\left(3 \mathrm{p}_{\pi}\right)^{1}\right]$ & 407.8 & $(400.8)$ & 394.4 & $(394.0)$ \\
\hline $\mathrm{R}_{3}\left(3 \mathrm{p}_{\sigma}\right)\left[(5 \sigma)^{2}(\pi)^{4}\left(\pi^{*}\right)^{1}\left(3 \mathrm{p}_{\sigma}\right)^{1}\right]$ & $(407.8)$ & $(400.8)$ & $(394.2)$ & $(394.2)$ \\
\hline $\mathrm{R}_{4}\left(3 \mathrm{p}_{\pi}\right)\left[(5 \sigma)^{2}(\pi)^{4}\left(\pi^{*}\right)^{1}\left(3 \mathrm{p}_{\pi}\right)^{1}\right]$ & $(407.9)$ & $(400.9)$ & 394.3 & 394.0 \\
\hline $\mathrm{R}_{5}(3 \mathrm{~s})\left[(5 \sigma)^{2}(\pi)^{4}\left(\pi^{*}\right)^{1}(3 \mathrm{~s})^{1}\right]$ & 408.0 & $(402.0)$ & 394.3 & $(395.1)$ \\
\hline $\mathrm{R}_{6}(3 \mathrm{~s})\left[(5 \sigma)^{2}(\pi)^{4}\left(\pi^{*}\right)^{1}(3 \mathrm{~s})^{1}\right]$ & $(408.2)$ & 402.2 & $(394.5)$ & $(395.3)$ \\
\hline $\mathrm{R}_{7}\left(3 \mathrm{p}_{\pi}\right)\left[(5 \sigma)^{2}(\pi)^{4}\left(\pi^{*}\right)^{1}\left(3 \mathrm{p}_{\pi}\right)^{1}\right]$ & $(408.2)$ & $(401.2)$ & $(394.6)$ & $(394.4)$ \\
\hline $\mathrm{R}_{8}\left(3 \mathrm{p}_{\pi}\right)\left[(5 \sigma)^{2}(\pi)^{4}\left(\pi^{*}\right)^{1}\left(3 \mathrm{p}_{\pi}\right)^{1}\right]$ & $(408.5)$ & $(401.6)$ & $(395.0)$ & $(394.6)$ \\
\hline $\mathrm{R}_{9}\left(3 \mathrm{p}_{\pi}\right)\left[(5 \sigma)^{2}(\pi)^{4}\left(\pi^{*}\right)^{1}\left(3 \mathrm{p}_{\pi}\right)^{1}\right]$ & $(409.1)$ & 402.1 & $(395.5)$ & $(395.3)$ \\
\hline $\mathrm{R}_{10}\left(3 \mathrm{p}_{\sigma}\right)\left[(5 \sigma)^{2}(\pi)^{4}\left(\pi^{*}\right)^{1}\left(3 \mathrm{p}_{\sigma}\right)^{1}\right]$ & $(409.1)$ & $(402.1)$ & $(395.5)$ & $(395.5)$ \\
\hline $\mathrm{R}_{11}\left(3 \mathrm{p}_{\sigma}\right)\left[(5 \sigma)^{2}(\pi)^{4}\left(\pi^{*}\right)^{1}\left(3 \mathrm{p}_{\sigma}\right)^{1}\right]$ & $(409.1)$ & $(402.1)$ & $(395.5)$ & $(395.5)$ \\
\hline $\mathrm{R}_{12}\left(3 \mathrm{p}_{\pi}\right)\left[(5 \sigma)^{2}(\pi)^{4}\left(\pi^{*}\right)^{1}\left(3 \mathrm{p}_{\pi}\right)^{1}\right]$ & $(409.2)$ & 402.2 & $(395.7)$ & $(395.4)$ \\
\hline \multicolumn{5}{|l|}{ Region iii } \\
\hline $\mathrm{D}_{1}\left[(5 \sigma)^{2}(\pi)^{3}\left(\pi^{*}\right)^{3}\right]$ & $(406.2)$ & & \multicolumn{2}{|c|}{ 397.4-398.4 } \\
\hline $\mathrm{D}_{2}\left[(5 \sigma)^{2}(\pi)^{3}\left(\pi^{*}\right)^{3}\right]$ & $(407.7)$ & & \multicolumn{2}{|c|}{ 398.8-399.9 } \\
\hline $\mathrm{D}_{3}\left[(5 \sigma)^{2}(\pi)^{3}\left(\pi^{*}\right)^{3}\right]$ & $(407.7)$ & & \multicolumn{2}{|c|}{ 398.8-399.9 } \\
\hline $\mathrm{D}_{4}\left[(5 \sigma)^{2}(\pi)^{3}\left(\pi^{*}\right)^{3}\right]$ & $(408.0)$ & & \multicolumn{2}{|c|}{$(399.1-400.2)$} \\
\hline
\end{tabular}


Finally, we discuss Region iii of the experimental map, which is shown in detail in the top right panel of Figure 1 (b). We find intensity predominantly at emission energies of 397.2 and 398.0 $\mathrm{eV}$, but also extending to slightly higher emission energies. In the excitation energy direction, the feature at $398.0 \mathrm{eV}$ emission energy shows distinct maxima at excitation energies of 405.6 and $407.7 \mathrm{eV}$, with the lower energy feature being particularly broad. The feature at $397.2 \mathrm{eV}$ emission energy has its first weak maximum at an excitation energy of $407.7 \mathrm{eV}$, with a long tail extending to lower excitation energies, and shows the most intense peak at an excitation energy of $408.7 \mathrm{eV}$. The assignment of the electronic transitions that lead to these features is not straightforward. Within a single electron excitation and emission framework there are no transitions predicted in this region, which indicates a more complex explanation for the origin of these features. In contrast, the simulated map does show peaks with significant intensity in this region, which arise from doubly excited states and correspond to $1 \mathrm{~s}_{\mathrm{N}} \rightarrow \pi^{*}$ excitation in combination with $\pi \rightarrow \pi^{*}$ excitations. In this case, the excitation step forms an intermediate state with a valence electronic configuration of $(5 \sigma)^{2}(\pi)^{3}\left(\pi^{*}\right)^{3}$ and a singly occupied $1 \mathrm{~s}_{\mathrm{N}}$ orbital. This corroborates the assignment of the weak features in the $397-400 \mathrm{eV}$ emission energy region in the non-resonant emission spectrum. The large number of possible doubly excited states makes a comprehensive account of all these states beyond the scope of our calculations, which are limited to the four lowest energy doubly excited states in this energy region (denoted $\mathrm{D}_{\mathrm{i}}$ in Table 1). Furthermore, emission from these states can lead to a variety of final states, and hence a range of energies is given in Table 1 to reflect this. 


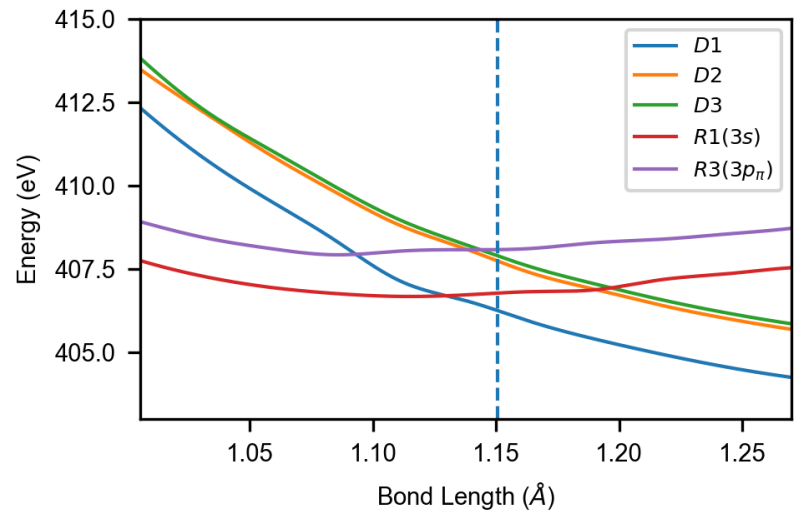

Figure 2. Calculated RASPT2 core-excited potential energy curves for the low-lying Rydberg and doubly excited states of NO. The vertical dashed blue line indicates the equilibrium ground state bond length.

It is important to note that the features in Region iii are responsible for a weak broad feature at $\sim 405 \mathrm{eV}$ in the nitrogen $\mathrm{K}$ XAS spectrum of $\mathrm{NO}$, the nature of which has been discussed in literature. In an early work, it was assigned to a dissociative $1 \mathrm{~s}_{\mathrm{N}} \rightarrow 2 \mathrm{p} \sigma^{*}$ transition. ${ }^{32}$ However, our calculations show that this transition lies at a significantly higher energy. In contrast and as discussed above, our simulations show doubly excited states as the origin of the feature at $405 \mathrm{eV}$ in the nitrogen $\mathrm{K} \mathrm{XAS}$, consistent with a recent theoretical study. ${ }^{42}$

While the simulated map in Figure 1 (c) qualitatively explains the presence of emission features in Region iii, the quantitative agreement is not very good. This can be attributed to the fact that the simulation only accounts for some of the possible doubly excited states. Furthermore, as will be discussed in the following, nuclear dynamics / vibronic coupling occurring on the timescale of the X-ray emission process also play a role. A study of the related $\mathrm{O}_{2}$ system has also highlighted the complexity of the electronic structure of the core-excited states for open-shell molecules. In 
particular, multiple crossings of potential energy curves were observed, and vibronic coupling was shown to influence the intensity of the calculated spectra. ${ }^{22}$ Figure 2 shows potential energy curves for the low-lying Rydberg and doubly excited states of NO. Similar to $\mathrm{O}_{2}$, multiple crossings between the curves for the different states are observed in the region of the equilibrium bond length, which suggests that non-adiabatic effects may play a role. Furthermore, the potential energy curves show that the doubly excited states are dissociative, caused by three electrons in the $\pi^{*}$ anti-bonding orbitals. We have explored the dissociative nature of the doubly excited states in more detail in the context of the vertical (excitation-energy) broadening of the feature in Region iii. Figure S4 in the Supporting Information compares the simulated RIXS maps with the equilibrium and an extended bond length, respectively. We find that the predicted bands arising from the $1 \mathrm{~s}_{\mathrm{N}} \rightarrow \pi^{*}$ and $1 \mathrm{~s}_{\mathrm{N}} \rightarrow$ Rydberg excitations are affected, but their energetic position only changes slightly. However, the features arising from the double excitations shift to significantly lower excitation energies, e.g., from 406.0 to $404.5 \mathrm{eV}$. This might explain the maxima observed at similar excitation energies in the experimental map and suggests that vibrational effects play an important role for these transitions.

In summary, the nitrogen K-edge RIXS map for the stable radical molecule NO has been discussed by comparing an experimental and a simulated map, the latter based upon RASSCF/RASPT2 calculations. The measured RIXS map shows a number of features that involve intermediate states arising from $1 \mathrm{~s}_{\mathrm{N}} \rightarrow \pi^{*}$ and $1 \mathrm{~s}_{\mathrm{N}} \rightarrow$ Rydberg excitations. Other distinct bands in the RIXS map cannot be rationalized within a one electron transition picture, but the simulations rather suggest that these features arise from doubly excited intermediate states comprising $1 \mathrm{~s}_{\mathrm{N}} \rightarrow \pi^{*}$ and $\pi \rightarrow \pi^{*}$ excitations. This is consistent with the observation of weak bands in the (simpler) non-resonant $\mathrm{x}$-ray emission 
spectrum, which can now also be attributed to double excitations. Nitric oxide is a major atmospheric pollutant which has led to interest in its catalytic reduction. This study demonstrates the value of combined experimental and theoretical studies of RIXS for revealing details of the complex electronic structure of radicals such as $\mathrm{NO}$, which can underpin fundamental studies of the catalytic process of NO reduction.

\section{Experimental Methods}

XES and RIXS experiments were carried out at beamline 8.0.1 of the Advanced Light Source (ALS), Lawrence Berkeley National Laboratory (Berkeley, USA) with the dedicated Solid And Liquid Spectroscopic Analysis (SALSA) roll-up experimental station. ${ }^{43} \mathrm{NO}$ gas from a gas bottle (purity 99.5\%) was introduced into and continuously pumped $(2 \mathrm{sccm})$ through a gas cell, separated from the ultra-high vacuum of the analysis chamber by a thin $\mathrm{SiC}$ membrane $(150 \mathrm{~nm}$

thick, NTT); it is described in more detail elsewhere. ${ }^{44}$ Spectra were collected using a hightransmission soft $\mathrm{x}$-ray spectrometer ${ }^{10}$ with a variable line space (VLS) grating and a combined (x-ray spectrometer and beamline) resolving power $E / \Delta E$ of better than 1000 . The excitation energy scale was calibrated by measuring the XAS spectrum of $\mathrm{N}_{2}$ gas. ${ }^{45}$ The emission energy scale was then adjusted using the elastically scattered (Rayleigh) lines, resulting in an uncertainty of the absolute energy scales of approximately $\pm 0.05 \mathrm{eV}$.

\section{Theoretical Methods}

The non-resonant $\mathrm{x}$-ray emission spectrum was simulated based upon EOM-CCSD calculations using a protocol described elsewhere. ${ }^{46,47,48}$ All states involved in the RIXS calculations have been 
calculated by the restricted active space self-consistent field (RASSCF) ${ }^{33}$ method, with the state energies determined using restricted active space second order perturbation theory (RASPT2). ${ }^{35,36}$ Transition dipole moments between states were computed using the restricted active space state interaction method (RASSI). ${ }^{49}$ All calculations were performed using the Molcas $8.2^{50}$ software package. Further details of the calculations are given in the Supporting Information.

\section{Supporting Information}

Further details of the calculations, simulated non-resonant x-ray emission spectra, and simulated RIXS map for an extended bond length.

\section{Acknowledgements}

The authors wish to thank the Leverhulme Trust for providing financial support (grant number RPG-2016-103). This research used resources of the Advanced Light Source, which is a DOE Office of Science User Facility under contract no. DE-AC02-05CH11231. AEAF also acknowledges support by the US Department of Energy, Office of Basic Energy Sciences, Division of Chemical Sciences, Geosciences, and Biosciences through Argonne National Laboratory under Contract No. DE-AC02-06CH11357. LS acknowledges support from the Helmholtz Association via Grant No. PD-326. The authors also wish to thank Doug Taube at the Lawrence Berkeley National Lab, without whom these experiments would not have been possible.

\section{References}

1. Bressler, C.; Chergui, M. Ultrafast X-ray Absorption Spectroscopy. Chem. Rev. 2004, $104,1781-1812$. 
2. Fuchs, O.; Zharnikov, M.; Weinhardt, L.; Blum, M; Weigand, M.; Zubavichus, Y.; Bär, M.; Maier, F.; Denlinger, J. D; Heske, C.; Grunze, M.; Umbach, E. Isotope and Temperature Effects in Liquid Water Probed by X-Ray Absorption and Resonant X-Ray Emission Spectroscopy. Phys. Rev. Lett. 2008, 100, 027801.

3. Weinhardt, L.; Weigand, M.; Fuchs, O.; Bär, M.; Blum, M.; Denlinger, J. D.; Yang, W.; Umbach, E.; Heske, C. Nuclear Dynamics in the Core-Excited State of Aqueous Ammonia Probed by Resonant Inelastic Soft X-ray Scattering. Phys. Rev. B 2011, 84, 104202.

4. Weinhardt, L.; Benkert, A.; Meyer, F.; Blum, M.; Wilks, R. G.; Yang, W.; Bär, M.; Reinert, F.; Heske, C. Nuclear Dynamics and Spectator Effects in Resonant Inelastic Soft X-ray Scattering of Gas-Phase Water Molecules. J. Chem. Phys. 2012, 136, 144311.

5. Chen, L. X.; Zhang, X.; Shelby, M. L. Recent Advances on Ultrafast X-ray Spectroscopy in the Chemical Sciences. Chem. Sci. 2014, 5, 4136-4152.

6. Chergui, M.; Collet, E. Photoinduced Structural Dynamics of Molecular Systems Mapped by Time-Resolved X-ray Methods. Chem. Rev. 2017, 117, 11025-11065.

7. Ament, L. J. P.; van Veenendaal, M.; Devereaux, T. P.; Hill, J. P.; van den Brink, J. Resonant Inelastic X-ray Scattering Studies of Elementary Excitations. Rev. Mod. Phys. 2011, 83, 705-767.

8. Saçek, P.; Carravetta, V.; Gel'mukhanov, F.; Ågren, H. Theory and First Principles Calculations of Dissociative Resonant Photoionization: The Evolution of Atomic Peaks and Holes. J. Chem. Phys. 2002, 116, 629-645.

9. Morin, P.; Miron, C. Ultrafast Dissociation: An Unexpected Tool for Probing Molecular Dynamics. J. Electron Spectrosc. Relat. Phenom. 2012, 185, 259 - 266. 
10. Fuchs, O.; Weinhardt, L.; Blum, M.; Weigand, M.; Umbach, E.; Bär, M.; Heske, C.; Denlinger, J.; Chuang, Y. D.; McKinney, W.; Hussain, Z.; Gullikson, E.; Jones, M.; Batson, P.; Nelles, B.; Follath, R. High-Resolution, High-Transmission Soft X-Ray Spectrometer for the Study of Biological Samples. Rev. Sci. Instrum. 2009, 80, 63103.

11. Weinhardt, L.; Fuchs, O.; Fleszar, A.; Bär, M.; Blum, M.; Weigand, M.; Denlinger, J. D.; Yang, W.; Hanke, W.; Umbach, E.; Heske, C. Resonant Inelastic Soft X-ray Scattering of CdS: A Two-Dimensional Electronic Structure Map Approach. Phys. Rev. B 2009, 79, 165305.

12. Couto, R.C.; Cruz, V.V.; Ertan, E.; Eckert, S.; Fondell, M.; Dantz, M.; Kennedy, B.; Schmitt, T.; Pietzsch, A.; Guimarães, F.F.; Ågren, H.; Gel'mukhanov, F.; Odelius, M.; Kimberg, V.; Föhlisch, A. Selective Gating to Vibrational Modes Through Resonant X-ray Scattering. Nat. Commun. 2017, 8, 14165.

13. Ertan, E.; Savchenko, V.; Ignatova, N.; Vaz da Cruz, V.; Couto, R. C.; Eckert, S.; Fondell, M.; Dantz, M.; Kennedy, B.; Schmitt, T.; Pietzsch, A.; Fhlisch, A.; Gel'mukhanov, F.; Odelius, M.; Kimberg, V. Ultrafast Dissociation Features in RIXS Spectra of the Water Molecule. Phys. Chem. Chem. Phys. 2018, 20, 14384-14397.

14. Fouda, A. E.; Purnell, G. I.; Besley, N. A. Simulation of Ultra-Fast Dynamics Effects in Resonant Inelastic X-ray Scattering of Gas-Phase Water. J. Chem. Theory Comput. 2018, $14,2586-2595$.

15. Glans, P.; Skytt, P.; Gunnelin, K.; Guo, J.-H.; Nordgren, J. Selectively Excited X-ray Emission Spectra of N $2 . J$. Electron Spectrosc. Relat. Phenom. 1996, 82, 193-201. 
16. Rubensson, J.-E.; Söderström, J.; Binggeli, C.; Gråsjö, J.; Andersson, J.; Såthe, C.; Hennies, F.; Bisogni, V.; Huang, Y.; Olalde, P.; Schmitt, T.; Strocov, V. N.; Föhlisch, A.; Kennedy, B.; Pietzsch, A. Rydberg-Resolved Resonant Inelastic Soft X-Ray Scattering: Dynamics at Core Ionization Thresholds. Phys. Rev. Lett. 2015, 114, 133001.

17. Skytt, P.; Glans, P.; Gunnelin, K.; Guo, J.; Nordgren, J.; Luo, Y.; Ågren, H. Role of Screening and Angular Distributions in Resonant X-ray Emission of CO. Phys. Rev. A 1997, $55,134-145$.

18. Couto, R.C.; Guarise, M.; Nicolaou, A.; Jaouen, N.; Chiuzbaian, G.S.; Lüning, J.; Ekholm, V.; Rubensson, J-E.; Såthe, C.; Hennies, F.; Guimarães, F.F.; Ågren, H.; Gel'mukhanov, F. and Journel, L.; Simon, M. and Kimberg, V. Coupled Electron-Nuclear Dynamics in Resonant $1 \sigma \rightarrow 2 \pi$ X-ray Raman Scattering of CO Molecules. Phys. Rev. A 2016, 93, 032510.

19. Glans, P.; Gunnelin, K.; Skytt, P.; Guo, J.-H.; Wassdahl, N.; Nordgren, J.; Ågren, H.; Gel'mukhanov, F.; Warwick, T.; Rotenberg, E. Resonant X-ray Emission Spectroscopy of Molecular Oxygen. Phys. Rev. Lett. 1996, 76, 2448-2451.

20. Adachi, J.; Kosugi, N.; Yagishita, A. Symmetry-Resolved Soft X-ray Absorption spectroscopy: Its Application to Simple Molecules. J. Phys. B: At. Mol. Opt. 2005, 38, R127-R152.

21. Pietzsch, A.; Sun, Y.-P.; Hennies, F.; Rinkevicius, Z.; Karlsson, H. O.; Schmitt, T.; Strocov, V. N.; Andersson, J.; Kennedy, B.; Schlappa, J.; Föhlisch, A.; Rubensson, J.-E.; Gel'mukhanov, F. Spatial Quantum Beats in Vibrational Resonant Inelastic Soft X-Ray Scattering at Dissociating States in Oxygen. Phys. Rev. Lett. 2011, 106, 153004. 
22. Feifel, R.; Velkov, Y.; Carravetta, V.; Angeli, C.; Cimiraglia, R.; Salek, P.; Gel'mukhanov, F.; Sorensen, S.L.; Piancastelli, M.N.; De Fanis, A.; Okada, K.; Kitajima, M.; Tanaka, T.; Tanaka, H.; Ueda, K. X-ray Absorption and Resonant Auger Spectroscopy of $\mathrm{O}_{2}$ in the Vicinity of the O 1s $\rightarrow \sigma^{*}$ Resonance: Experiment and Theory. J. Chem. Phys. 2008, 128, 064304.

23. Nagano, T.; Yoshimura, T. Bioimaging of Nitric Oxide. Chem. Rev. 2002, 102, 1235-1270.

24. de Vivie, R.; Peyerimhoff, S. D. Theoretical Spectroscopy of the NO Radical. I. Potential Curves and Lifetimes of Excited States. J. Chem. Phys. 1988, 89, 3028- 3043.

25. Shi, H.; East, A. L. L. Improved Results for the Excited States of Nitric Oxide, Including the B/C Avoided Crossing. J. Chem. Phys. 2006, 125, 104311.

26. Ershova, O. V.; Besley, N. A. Theoretical Calculations of the Excited State Potential Energy Surfaces of Nitric Oxide. Chem. Phys. Lett. 2011, 513, 179-183.

27. Tronc, M.; King, G. C.; Read, F. H. Nitrogen K-shell Excitation in $\mathrm{N}_{2}$, NO and $\mathrm{N}_{2} \mathrm{O}$ by High-Resolution Electron Energy-Loss Spectroscopy. J. Phys. B: At. Mol. Opt. 1980, 13, 999.

28. Camilloni, R.; Fainelli, E.; Petrocelli, G.; Stefani, G. Generalised Oscillator Strength for the Core Level Excitation of Nitrogen in $\mathrm{N}_{2}, \mathrm{NO}$ and $\mathrm{N}_{2} \mathrm{O}$ by Electron Impact. J. Phys. $B$ : At. Mol. Opt. 1987, 20, 1839.

29. Remmers, G.; Domke, M.; Puschmann, A.; Mandel, T.; Kaindl, G.; Hudson, E.; Shirley, D. High-Resolution Inner-Shell Photoionization of NO. Chem. Phys. Lett. 1993, 214, 241249.

30. Fink, R. A Theoretical Simulation of the $1 \mathrm{~s} \rightarrow 2 \pi$ Excitation and Deexcitation Spectra of the NO Molecule. J. Chem. Phys. 1997, 106, 4038-4052. 
31. Püttner, R.; Dominguez, I.; Morgan, T.; Cisneros, C.; Fink, R.; Rotenberg, E.; Warwick, T.; Domke, M.; Kaindl, G.; Schlachter, A. Vibrationally Resolved O 1s Core-Excitation Spectra of CO and NO. Phys. Rev. A 1999, 59, 3415.

32. Wight, G.R.; Brion, C.E. K-Shell Excitations in $\mathrm{NO}$ and $\mathrm{O}_{2}$ by $2.5 \mathrm{keV}$ Electron Impact. $J$. Electron Spectrosc. Relat. Phenom. 1974, 4, 313-325.

33. Fukuzawa, H.; Liu, X.-J.; Montuoro, R.; Lucchese, R. R.; Morishita, Y.; Saito, N.; Kato, M.; Suzuki, I. H.; Tamenori, Y.; Teranishi, T.; Lischke, T.; Promper, G.; Ueda, K. Nitrogen K-shell Photoelectron Angular Distribution From NO Molecules in the Molecular Frame. J. Phys. B: At. Mol. Opt. 2008, 41, 045102.

34. Demekhin, P. V.; Petrov, I. D.; Sukhorukov, V. L.; Kielich, W.; Knie, A.; Schmoranzer, H.; Ehresmann, A. Strong Interference Effects in the Angularly Resolved Auger Decay and Fluorescence Emission Spectra of the Core-Excited NO Molecule. J. Phys. B: At. Mol. Opt. 2010, 43, 165103.

35. Malmqvist, P.-Å.; Rendell, A.; Roos. B.O. The Restricted Active Space Self-ConsistentField Method, Implemented with a Split Graph Unitary Group Approach. J. Phys. Chem., 1990, $94,5477-5482$.

36. Andersson, K.; Malmqvist, P.-Å.; Roos, B.O. Second-Order Perturbation Theory with a Complete Active Space Self-Consistent Field Reference Function. J. Chem. Phys. 1992, 96, 1218-1226.

37. Kramers, H. A.; Heisenberg, W. Über Die Streuung von Strahlung Durch Atome. Zeitschrift für Physik A Hadrons and Nuclei 1925, 31, 681-708.

38. Gel'mukhanov, F.; Ågren, H. Resonant Inelastic X-ray Scattering with SymmetrySelective Excitation. Phys. Rev. A 1994, 49, 4378-4389. 
39. Luo, Y.; Ågren, H.; Gel'mukhanov, F. Symmetry Assignments of Occupied and Unoccupied Molecular Orbitals Through Spectra of Polarized Resonance Inelastic X-ray Scattering. J. Phys. B-At. Mol. Opt. 1994, 27, 4169.

40. Luo, Y.; Ågren, H.; Gel'mukhanov, F. Polarization Anisotropy in Resonant X-ray Emission from Molecules. Phys. Rev. A 1996, 53, 1340.

41. Rubensson, J.-E.; Petersson, L.; Wassdahl, N.; Bäckström, M.; Nordgren, J. Radiative Decay of Multiply Excited Core Hole States in $\mathrm{H}_{2} \mathrm{O}$. J. Chem. Phys. 1985, 82, 4486-4491.

42. Oosterbaan, K.J.; White, A.F.; Head-Gordon, M. Non-Orthogonal Configuration Interaction with Single Substitutions for Core-Excited States: An Extension to Doublet Radicals. J. Chem. Theory Comput. 2019, 15, 2966-2973.

43. Blum, M.; Weinhardt, L.; Fuchs, O.; Bär, M.; Zhang, Y.; Weigand, M.; Krause, S.; Pookpanratana, S.; Hofmann, T.; Yang, W.; Denlinger, J. D.; Umbach, E.; Heske, C. Solid and Liquid Spectroscopic Analysis (SALSA) - A Soft x-Ray Spectroscopy Endstation with a Novel Flow-through Liquid Cell. Rev. Sci. Instrum. 2009, 80, 123102.

44. Benkert, A.; Blum, M.; Meyer, F.; Wilks, R. G.; Yang, W.; Bär, M.; Reinert, F.; Heske, C.; Weinhardt, L. Setup for in Situ Investigation of Gases and Gas/Solid Interfaces by Soft X-Ray Emission and Absorption Spectroscopy. Rev. Sci. Instrum. 2014, 85, 01511.

45. Kato, M.; Morishita, Y.; Oura, M.; Yamaoka, H.; Tamenori, Y.; Okada, K.; Matsudo, T.; Gejo, T.; Suzuki, I. H.; Saito, N. Absolute Photoionization Cross Sections with Ultra-High Energy Resolution for Ar, $\mathrm{Kr}, \mathrm{Xe}$ and $\mathrm{N}_{2}$ in Inner-Shell Ionization Regions. J. Electron Spectrosc. Relat. Phenom. 2007, 16, 39-48. 
46. Besley, N.A. Equation of Motion Coupled Cluster Theory Calculations of the X-ray Emission Spectroscopy of Water. Chem. Phys. Lett. 2012, 542, 42-46.

47. Wadey, J.D.; Besley, N.A. Quantum Chemical Calculations of X-ray Emission Spectroscopies. J. Chem. Theory Comput. 2014, 10, 4557-4564.

48. Fouda, A.E.A.; Besley, N.A. Assessment of Basis Sets for Density Functional Theory Based Calculations of Core Electron Spectroscopies. Theor. Chem. Acc. 2018, 137, 6.

49. Malmqvist, P.-Å.; Roos, B.O. The CASSCF State Interaction Method. Chem. Phys. Lett. 1989, 24, 189-194.

50. Aquilante, F.; Autschbach, J.; Carlson, R.K.; Chibotaru, L.F.; Delcey, M.G.; De Vico, L.; Fdez, G.I.; Ferré, N.; Frutos, L.M.; Gagliardi, L.; Garavelli, M.; Giussani, A.; Hoyer, C.E.; Li Manni, G.; Lischka, H.; Ma, D.; Malmqvist, P.Å.; Müller, T.; Nenov, A.; Olivucci, M.; Pedersen, T.B.; Peng, D.; Plasser, F.; Pritchard, B.; Reiher, M.; Rivalta, I.; Schapiro, I.; Segarra-Martí, J.; Stenrup, M.; Truhlar, D.G.; Ungur, L.; Valentini, A.; Vancoillie, S.; Veryazov, V.; Vysotskiy, V.P.; Weingart, O.; Zapata, F.; Lindh, R. Molcas 8: New Capabilities for Multiconfigurational Quantum Chemical Calculations Across the Periodic Table. J. Comput. Chem. 2016, 37, 506-541. 